\title{
Für ein menschenwürdiges Dasein «intersexueller» Personen
}

Heike Walz*

* Juniorprofessorin für Feministische Theologie und theologische Frauenforschung an der Kirchlichen Hochschule Wuppertal/Bethel mit den Schwerpunkten Geschlechterforschung, Menschenrechte; bis April 2009 Dozentin für Systematische Theologie am I.U. ISEDET in Buenos Aires (als ökumenische Mitarbeiterin von mission 21 Basel); Pfarrerin; Mitbegründerin des Netzwerkes Geschlechterbewusster Theologie (NGT) in der Schweiz, Deutschland und Österreich.

Korrespondenz:

Dr. theol. Heike Walz Kirchliche Hochschule Wuppertal/Bethel Arbeitsbereich Wuppertal Missionsstr. 9a/b D-42285 Wuppertal

heike.walz@gmx.ch

\section{Wie fühlen sich Menschen mit uneindeutigem Geschlecht?}

Wie Menschen sich selbst fühlen, wenn sie anatomisch nicht eindeutig als weiblich oder männlich zu identifizieren sind, war bis vor kurzem in der Öffentlichkeit eher ein Tabu. In der Schweiz kam 2008 der Film «XXY» (Argentinien, Spanien, Frankreich 2007) der argentinischen Regisseurin und Schriftstellerin Lucía Puenzo in die Kinos: Alex, fünfzehn Jahre alt, ist mit den Eltern in ein abgelegenes Haus an der Küste in Uruguay umgezogen. Doch auch hier kann sie (von den Eltern als Mädchen erzogen) den Hänseleien aufgrund ihres geschlechtlich unentschiedenen Körpers nicht entgehen. Sie möchte von den anderen akzeptiert werden, einfach sie selbst sein. Aber wer ist sie? Am liebsten wäre ihr, alles würde gleichbleiben: Keine Hormone mehr, keine Umzüge und vor allem keine Operation ... Der Film [1] zeigt auf eindrückliche Weise, wie Alex fühlt und lebt, und dass öffentliche Aufklärung über «Intersexualität» dringend nötig ist.

\section{Um was geht es in diesem Band?}

Hierzu leistet der sehr lesenswerte Sammelband einen wichtigen Beitrag. Er dokumentiert die durchweg spannenden Vorträge, die am 17. Juli 2004 in einem Kolloquium an der Universität Fribourg diskutiert wurden. Anstelle eines Patentrezepts will der Band einen Diskussionsbeitrag bieten, der den Beteiligten Betroffenen, Eltern, Ärzten, Psychologinnen, Sozialarbeitern, Hebammen - bei den zu treffenden ethischen Entscheidungen eine Orientierungshilfe bietet (S. 8). Dies gelingt durch die «transdisziplinäre» Herangehensweise, bei der die Stimmen der Betroffenen zu Wort kommen und fachwissenschaftliche Beiträge aus den Sozialwissenschaften, Philosophie, Medizin, Psychologie und Rechtswissenschaften einbezogen werden. Standen diese Disziplinen bislang kaum in Dialog über «Intersex», ist in diesem Band spürbar, wie sie wechselseitig Gedanken aufnehmen. Die gelungene Verzahnung zwischen Wissenschaft und ethischer Handlungsorientierung zeigt sich auch am Schluss des Bandes: Die Empfehlungen für den Umgang mit «intersexuellen» Kindern wurden mit Selbsthilfegruppen besprochen und bündeln die Erkenntnisse der Beiträge im Buch. Hilfreich sind auch das Glossar der Fachtermini und die Kontaktadressen.

\section{Zum Begriff «Intersex»}

Wie sehr die Debatten über «Intersexualität» im Fluss sind, zeigt sich bereits an der Terminologie: Es besteht kein Konsens, auch nicht unter den Betroffenen. Während sich die Fachdisziplinen 2005 auf DSD-Syndrome (Disorders of Sex Development) einigten, schlägt Mirjam Werlen biologisches Geschlechtsvariationssyndrom (BGV) vor. Die Herausgebenden bleiben bei dem Begriff Intersex. Dies leuchtet insofern ein, als Menschen so nicht mit Kürzeln bezeichnet werden und neben gesundheitlichen und körperlichen Aspekten auch soziale Folgen anklingen. Der Band wahrt die terminologische Pluralität, indem jede Autorin ihre Begriffe selbst wählt.

\section{Die Stimme von Betroffenen hören}

Das subjektive Erleben von «Intersex» mit der Wissenschaft in Kontakt zu bringen, ist ein roter Faden des Bandes. Karin Plattner, Mutter eines Kindes mit unklarem Geschlecht, gründete in der Schweiz den Verein SI Selbsthilfe Intersexualität. Anstatt «Intersex» als Störung wahrzunehmen, hat sie das positive Potential sehen gelernt. Sie träumt davon, dass Kinder von klein auf lernen, dass das «Dazwischen», die Vielfalt der Intersexualität, normal ist. Evelines Schilderung ihres Lebenskampfes mit «Intersex» sei als persönliche Lektüre dringend empfohlen. Anhand einer sozialwissenschaftlichen Analyse von Erfahrungsberichten im Internet legt Kathrin Zehnder dar, dass Selbsthilfegruppen als Ressource erlebt werden, während im Kontakt mit der Familie und Ärzten (mit Ausnahme von Psychotherapeutinnen) meist Sprachlosigkeit herrscht. Die Potentiale der Sozialarbeit liessen sich hier weiter ausschöpfen. Bei «Intersex» handelt es sich somit keinesfalls um ein rein chirurgisches Problem, sondern um schwierige ethische Entscheidungen: Soll die Uneindeutigkeit des Geschlechts des Kindes so gelassen werden - oder ist das Kind zu schützen vor dem Gespött anderer? Wie wird das Kind später Operationen ohne sein Einvernehmen - oder eine Nichtbehandlung - beurteilen?

\section{Die Einzigartigkeit und das «Dazwischen» von «Intersex» respektieren}

Auch wenn der Band kein Patentrezept geben will, ist eine gemeinsame Richtung erkennbar: Ein «menschenwürdiges Dasein für Intersexuelle» (Groneberg, S. 141) auf der Basis der Menschenrechte bzw. dem Recht des Kindes auf Selbstbestimmung. Die Würde der Betroffenen zu respektieren, Informationen vollumfänglich zugänglich zu machen, «Intersex» zu enttabuisieren und die Normen hinsichtlich des Geschlechts zu erweitern: Dies ist nicht nur eine Herausforderung für die Ärzteschaft, sondern für die Gesellschaft insge- 


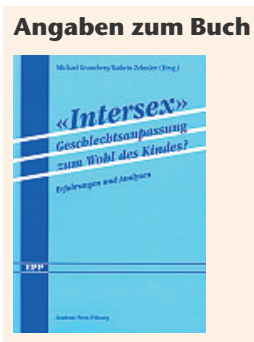

Titel: «Intersex». Geschlechtsanpassung zum Wohl des Kindes? Untertitel: Erfahrungen und Analysen

Reihe: Band 12 der Reihe Ethik und politische Philosophie, hrsg. vom Interdisziplinären Institut für Ethik und Menschenrechte an der Universität Freiburg Schweiz

Verlag: Freiburg i.Ü.: Academic Press Fribourg/Paulusverlag: 2008

ISBN: 978-3-7278-1506-5

Preis: 46 Franken

Herausgeber:

Michael Groneberg ist promovierter und habilitierter Hochschullehrer und -forscher in Philosophie (Ethik, Wissenschaftsphilosophie, Antike); Kathrin Zehnder ist lizenzierte Sozialwissenschafterin, diplomierte Sozialarbeiterin und promoviert im Fach Soziologie im Rahmen eines vom Schweizerischen Nationalfonds geförderten Pro*Doc-Projekts.

Autoren und Autorinnen:

Eveline (AGS-Betroffene),

Michael Groneberg (Philoso-

phie), Karin Plattner (Mutter),

Hertha Richter-Appelt (Sexualwissenschaft), Jürg Rieben (Psychologie), Mirjam Werlen

(Recht), Kathrin Zehnder (Sozialwissenschaft). samt. Konkret bedeutet dies, die endgültige Festlegung des Geschlechts offenzuhalten, da die Selbstidentifikation als Mädchen oder Junge nicht vor dem Alter von 2 bis 3 Jahren entsteht. Zwar wird zur Wahl eines «Erziehungsgeschlechts» geraten, aber im Zentrum steht, das «eigentliche» Geschlecht der Person zu respektieren - gerade auch dann, wenn dies von rein weiblichen oder männlichen Stereotypen abweicht. Die Einzigartigkeit und Selbstbestimmung des Kindes soll Vorrang haben vor soziokulturellen Geschlechtsnormen, elterlichen Präferenzen oder medizinischen Behandlungsmustern.

Michael Groneberg legt in seiner profunden philosophischen Untersuchung den geschichtlichen Wandel der Interpretationen von Intersexualität (von Platon bis Freud) dar, und zwar ausgehend von den Mythen der abendländischen Philosophie und der jüdischchristlichen Tradition. Während beispielsweise das mittelalterliche kanonische Recht vorsah, dass Intersexuelle als Erwachsene ihr Geschlecht wählen, setzt im 18. Jahrhundert die Medizin ein «wahres Geschlecht» fest. Angesichts der Vielfalt der Kriterien zur Feststellung des Geschlechts zeigt er, dass die Anatomie nicht ausreicht. Es geht um die «geschlechtliche[n] Verfasstheit» (S. 139) der Person. Für die Theoriebildung der Geschlechterforschung ist interessant, dass er infolgedessen über die Unterscheidung von biologischem Geschlecht (sex), sozialem Geschlecht (gender) und Sexualität (desire) hinausgeht und den Begriff der psychischen Geschlechtsidentität einführt. Für den gesamten Band ist die systematische Unterscheidung zwischen biologischem Geschlecht, Zuweisungsgeschlecht (Eintrag ins Register), Erziehungsgeschlecht, später entstehender sozialer Geschlechtsrollenidentität und psychischer Geschlechtsidentität wegweisend. Hier schliesst Jürg Rieben an, der den psychologischen Begriff der Geschlechtsidentität als individuellen, lebenslangen (und nicht einen auf die beiden ersten Lebensjahre begrenzten) Prozess charakterisiert.

\section{Medizin und Recht vor neuen Fragen}

Hertha Richter-Appelt kommt in ihrer Analyse der weltweit bislang einzigartigen Hamburger Studie zu erwachsenen Personen mit Intersexualität zu dem Schluss, dass zwischen den verschiedenen Diagnosegruppen deutlicher unterschieden werden sollte, vor allem zwischen Personen, die bereits nach der Geburt oder erst in der Pubertät Auffälligkeiten zeigen. Auf Operationen von Neugeborenen wirft sie neues Licht, da Betroffene sich als «zwangstranssexualisiert» erleben (S. 77). Die übliche Geheimhaltung der Diag- nose innerhalb und ausserhalb der Familie erweist sich, so Richter-Appelt, als kontraproduktiv, da sie zu Scham- und Schuldgefühlen, niedrigem Selbstwertgefühl und sozialer Isolation führe. Bislang sei der traumatisierende Aspekt geschlechtskorrigierender Operationen stark unterschätzt worden. Das Dilemma sei allerdings, dass das Wissen über Behandlungsoptionen und deren Langzeitfolgen unzureichend ist. Hier bestehe Bedarf an lebenslangen Studien.

Mirjam Werlen zeigt auf, dass das schweizerische Recht in Übereinstimmung mit der Kinderrechtskonvention (KRK) dem Grundsatz folgt: «Im Zweifel für die Kindesinteressen.» Steht das Recht auf Selbstbestimmung über die eigene Geschlechtsidentität im Zentrum, müssen ihrer Meinung nach chirurgische Eingriffe medizinisch zwingend indiziert sein, während kosmetische Operationen die Urteilsfähigkeit des Kindes ( $a b$ dem dritten bis sechsten Lebensjahr) abwarten sollten. So lange unklar sei, welche Langzeitfolgen frühzeitige Operationen für Kinder haben (namhafte Fachleute empfehlen ein Moratorium), empfiehlt sie die Begleitung durch eine Kommission aus Endokrinologen, Kinderärztinnen, Psychologen, Eltern und betroffenen Erwachsenen mit intersexuellen Kindern. Das Geschlecht eines intersexuellen Kindes sollte im Zivilstandsregister unter Vorbehalt einer späteren Ergänzung eingetragen werden können.

Der Band hält, was er verspricht: Stoff zur Diskussion bietet er in Fülle. Bei mir hat er die Frage geweckt, wie es Ärzte und Ärztinnen mit der ethischen Entscheidungsfindung und der Behandlung von Intersexuellen ergeht. Auch könnte in zukünftigen Debatten die Biologie (vgl. Gronebergs Beitrag) stärker einbezogen werden. Sie spielt im Alltagswissen eine wichtige Rolle. Da Religion und Kirche den Geschlechterdualismus religiös überhöhen und Betroffene berichten, wie die (Inter-)Sexualität in ihrem katholischen oder gläubigen Elternhaus als Tabu behandelt wurde (vgl. Zehnder), wäre ein Blick auf neuere theologische Ansätze hilfreich [2]. Kurzum: Nicht Intersexuelle sind ein Problem - sondern die Gesellschaft. Es gibt noch viel zu tun.

\section{Literatur}

1 Der Filmtitel «XXY» ist leicht irreführend, da Alex' «Intersex» eigentlich nicht dem Karyotyp XXY (Klinefelter Syndrom) entspricht, sondern dem Adrenogenitalen Syndrom (AGS), vgl. zu beiden Typen das Glossar S. 224-39.

2 Walz H, Plüss D (Hrsg.). Theologie und Geschlecht. Dialoge querbeet. Münster: LIT; 2008. 